\title{
Initiation Sequence of E-Cigarette and Cigarette Smoking among US Adolescents: A National Study
}

\author{
Sean Esteban McCabe, PhD ${ }^{1},{ }^{1,2}$ Philip Veliz, PhD, ${ }^{1,2}$ Vita V. McCabe, MD, ${ }^{3}$ Carol J. Boyd, PhD ${ }^{1,2,4}$ \\ ${ }^{1}$ Center for the Study of Drugs, Alcohol, Smoking, and Health, Department of Health Behavior and Biological Sciences, School of \\ Nursing, University of Michigan, Ann Arbor, Michigan \\ ${ }^{2}$ Institute for Research on Women and Gender, University of Michigan, Ann Arbor, Michigan \\ ${ }^{3}$ Lung Care and Smoking Cessation Program, St. Joseph Mercy Health System, Ypsilanti, Michigan \\ ${ }^{4}$ Addiction Center, Department of Psychiatry, University of Michigan, Ann Arbor, Michigan
}

Background and Objectives: The primary objectives were to: (1) examine the initiation sequence of e-cigarette use and cigarette smoking; (2) assess other substance use as a function of the initiation sequence of e-cigarettes and cigarettes; and (3) investigate the role of early e-cigarette initiation among US secondary school students.

Methods: Data were collected via self-administered questionnaires from independent 2015 and 2016 nationally representative cross-sectional samples of 8th grade, 10th grade, and 12th grade students $(N=36410)$. Results: The lifetime initiation sequence included: (1) e-cigarette use before cigarette smoking (1.7\%); (2) e-cigarette use and cigarette smoking in same grade $(4.2 \%)$; (3) cigarette smoking before e-cigarette use $(6.1 \%)$; (4) e-cigarette use only (12.6\%); (5) cigarette smoking only (3.6\%); and (6) no e-cigarette use or cigarette smoking $(71.8 \%)$. The risk of substance use was highest among dual users (regardless of initiation sequence), followed by cigarette smokers, e-cigarette users, and no e-cigarette use or cigarette smoking. The most prevalent initiation sequence of e-cigarette use involved initiating other substances before e-cigarettes, especially among older adolescents. Early initiation of e-cigarette use was associated with increased odds of substance use behaviors for all three age groups.

Discussion and Conclusions: Adolescents who report a history of both e-cigarette use and cigarette smoking should be considered at high risk for substance-related problems. Early initiation of e-cigarette use is a signal for other substance use.

Scientific Significance: Youth substance use prevention programs and prospective studies must take into account polysubstance use when addressing the relationships between e-cigarette use and cigarette smoking. (Am J Addict 2019;28:285-294)

\section{INTRODUCTION}

E-cigarettes are battery-powered vaporizer devices that may contain nicotine, flavorings or both nicotine and flavorings. The relatively high prevalence and rapid escalation in

Received October 4, 2018; revised February 2, 2019; accepted March 9, 2019.

Address correspondence to: Dr McCabe, $\mathrm{PhD}$, Center for the Study of Drugs, Alcohol, Smoking, and Health, School of Nursing, University of Michigan, 400N. Ingalls Street, Ann Arbor, MI 48109. E-mail: plius@umich.edu adolescents' e-cigarette use is a major public health concern, with more than one in every three US high school seniors reporting past-year use. ${ }^{1-4}$ The US has banned the sale of e-cigarettes to anyone under the age of 18 and the Food and Drug Administration is currently considering policies and strategies to further limit the sales of e-cigarette/vaping devices to youth. Several US cities and states have enacted laws that increased the legal age to purchase e-cigarettes to age 21. Yet despite the public concern over adolescents' use of e-cigarettes, not enough is understood about the relationship between e-cigarette use and cigarette smoking among adolescents. Indeed, an important question remains as to the initiation sequence involving e-cigarette use and cigarette smoking. There is evidence that adolescents are more likely to engage in cigarette smoking after using e-cigarettes than their peers who never initiated e-cigarette use. ${ }^{5-11}$ There is also research indicating that a large proportion of adolescents engage in dual use of e-cigarettes and cigarette smoking, with little attention to whether e-cigarette use preceded cigarette smoking or vice versa.

There is growing evidence that dual users have significantly greater risk of other substance use behaviors relative to those who only use e-cigarettes or smoke cigarettes. ${ }^{12-15}$ To date, prospective studies of e-cigarette use by adolescents have varied greatly in their inclusion of other substance use. In fact, it is not only cigarette smoking that is associated with e-cigarette use: "vaping" marijuana oil and marijuana use is also associated with e-cigarette use. ${ }^{16-18} \mathrm{At}$ least one study found marijuana use served as a significant pathway in the relationship between e-cigarette use and cigarette smoking onset. ${ }^{18}$ In a recent review, the inclusion of other substance use was uneven across studies and the mean age of six of the nine prospective studies of e-cigarette use and subsequent cigarette smoking focused on older adolescents aged 18 or older at baseline. ${ }^{19}$ Moreover, several studies combine a wide range of ages, but do not allow for a focus on different developmental points, particularly relative 
to the initiation of their e-cigarette use in relation to other substance use such as marijuana use. The initiation patterns of substance use are likely to change over time as adolescents become more heavily substance-involved from early adolescence to late adolescence. ${ }^{20}$

Some researchers have asserted that youth who initiate e-cigarette use at an early age are more likely to progress to cigarette smoking and other substances. ${ }^{12,18}$ However, there has been very little research examining the initiation patterns of e-cigarette and combustible cigarette use in early adolescence versus late adolescence. As a result, it remains unknown whether it is more common for younger (versus older) adolescents to initiate e-cigarette use before cigarette smoking or vice versa, and how initiation sequences are associated with other forms of substance use. To advance our understanding of adolescents' use of e-cigarettes, we proposed three main research objectives among US secondary school students: (1) to examine the initiation sequence of e-cigarette use and cigarette smoking; (2) to assess substance use behaviors as a function of the initiation patterns of e-cigarette use and cigarette smoking, and (3) to investigate the role of early initiation of e-cigarette use. Five initiation patterns were examined: (1) e-cigarette use before cigarette smoking, (2) cigarette smoking before e-cigarette use, (3) e-cigarette use and cigarette smoking in the same grade, (4) e-cigarette use only, and (5) cigarette smoking only.

\section{METHODS}

\section{Study Design}

The present study used national data from the Monitoring the Future (MTF) study, which annually surveys a cross-sectional, nationally representative sample of 8th, 10th, and 12th grade students attending US public and private secondary schools, using self-administered paper-and-pencil questionnaires in classrooms. The MTF study used a multistage sampling procedure. Stage 1 was the selection of geographic areas within the four regions of the country including the Northeast, South, Midwest, and West. Stage 2 was the random selection of public and private secondary schools. Stage 3 was the selection of students within each school. Corrective weighting was used in the analyses presented in this study to account for the unequal probabilities of selection that occurred at any stage of sampling. The samples analyzed in this study consisted of 8th, 10th, and 12th grade students from the 2015 (total sample, $n=17$ 892; 8th grade sample, $n=7518 ; 10$ th grade sample, $n=8075 ; 12$ th grade sample, $n=2299$ ) and 2016 (total sample, $n=18518$; 8th grade sample, $n=8810$; 10th grade sample, $n=7608 ; 12$ th grade sample, $n=2100$ ) cohorts (overall sample, $n=36410$ ). The response rates in the MTF study for the 2015 and 2016 cohorts were as follows: 8th grade students (89\% and 90\%), 10 th grade students (87\% and 88\%), and 12th grade students (83\% and $80 \%$ ). Additional details about the MTF design and methods are available elsewhere. ${ }^{2}$ Institutional Review Board approval was granted for this study by the University of Michigan Institutional Review Board.

\section{Measures}

The MTF study assesses a wide range of demographic characteristics and substance use behaviors, including ecigarette use and cigarette smoking. Demographic characteristics included sex, grade level (8th, 10th, and 12th), race/ethnicity (Black, White, Hispanic, and Other), US Census geographical region (Northeast, Midwest, South, and West), metropolitan statistical area (MSA) (large, other, and nonMSA), parental education (some college vs high school or less), and college plans (any plans to attend college vs no plans to attend college). These demographic characteristics were included in the multivariate analyses to account for potentially confounding factors that are known to be associated with cigarette smoking, e-cigarette use, and other substance use behaviors within the MTF sample. ${ }^{1-20}$

E-cigarette use was assessed by asking respondents on how many occasions, if any, they had used e-cigarettes in their lifetime. The lifetime response options ranged from (1) "Never" to (5) "Regularly." Cigarette smoking was assessed by asking respondents how often, if ever, they had smoked cigarettes in their lifetime. The lifetime response options ranged from (1) "Never" to (5) "Regularly."

Grade of initiation for e-cigarette use and cigarette smoking was assessed by asking respondents what grade level they first used either substance. The response options ranged from (1) "Never" to (6) "Grade 8" for respondents in 8th grade (ie, "Never," "Grade 4 or lower," "Grade 5" ... "Grade 7," "Grade 8"); (1) "Never" to (8) "Grade 10" for respondents in 10th grade (ie, "Never," "Grade 4 or lower," "Grade 5" ... "Grade 9," "Grade 10"); and (1) "Never" to (10) "Grade 12" for respondents in 12th grade (ie, "Never," "Grade 6 or lower," "Grade 7" ... "Grade 11," "Grade 12"). Initiation sequence of cigarette smoking and e-cigarette use initiation were determined by placing responses to the e-cigarette use items into one of five mutually exclusive categories: (1) e-cigarette use before cigarette smoking, (2) cigarette smoking before e-cigarette use, (3) e-cigarette use and cigarette smoking in the same grade, (4) e-cigarette use only, and (5) cigarette smoking only.

Alcohol, marijuana, and other illicit drug use was assessed by asking respondents on how many occasions, if any, they had been drunk or used marijuana, cocaine, lysergic acid diethylamide (LSD), psychedelics other than LSD, and heroin in their lifetimes. Nonmedical prescription drug misuse was assessed by asking respondents on how many occasions, if any, they used each prescription drug class (opioids, sedatives, stimulants, and tranquilizers) on their own without a doctor's prescription in their lifetime. The response scale for each substance ranged from (1) "No occasions" to (7) "40 or more occasions." Grade of initiation for alcohol, marijuana, and other substances was assessed in the identical manner as e-cigarette use and cigarette smoking. 


\section{Statistical Analysis}

All analyses for this study were design-based in nature, fully accounting for the MTF sampling weights in estimation of parameters for the target MTF population and incorporating estimates of MTF design effects (reflecting the complex sample design features) in variance estimates, confidence intervals, and test statistics. ${ }^{2}$ We initially computed descriptive estimates of selected parameters representing the prevalence of particular behaviors (eg, lifetime e-cigarette use) and then compared different subgroups of individuals based on the initiation patterns of ecigarette use and cigarette smoking in terms of the prevalence of each other substance use behavior. All variance estimates for the estimated descriptive parameters and the designadjusted test statistics incorporated an MTF average design effect of 2.5 for these specific types of behaviors (due to the MTF not providing variables that account for the complex sampling design). ${ }^{2}$ Next, multivariate logistic regression models were fitted using design-based approaches to estimate the associations of initiation patterns of e-cigarette and cigarette smoking with each behavior after adjusting for cohort, relevant sociodemographic characteristics, and other covariates examined in prior work. ${ }^{1-20}$ Next, we estimated the same models discussed above among lifetime e-cigarette users only and included additional covariates to account for grade of initiation of e-cigarette use. It should be noted that all analyses used the "svy" procedures in the Stata software (Version 14) that incorporated the weights provided in the public use MTF data files and incorporated design effects using the methodology of West and McCabe. ${ }^{21}$

\section{RESULTS}

Approximately three in every ten US secondary school students reported lifetime e-cigarette use. As shown in Table 1,

TABLE 1. Lifetime initiation sequence of e-cigarette use and cigarette smoking among US secondary school students, 2015 and 2016

Initiation sequence of e-cigarette use and cigarette smoking

Lifetime e-cigarette use and cigarette smoking $\%, N$

8th, 10th and 12th grade students

Initiated e-cigarette before cigarettes

$1.7,559$

Initiated e-cigarettes and cigarettes in same grade

$4.2,1334$

Cigarettes before e-cigarettes

$6.1,1955$

E-cigarette only

$12.6,4053$

Cigarettes only

3.6, 1116

Never used e-cigarette or cigarettes

Total, $N$

$71.8,22577$

31594

8th grade students

Initiated e-cigarette before cigarettes

$1.0,148$

Initiated e-cigarettes and cigarettes in same grade

Cigarettes before e-cigarettes

$2.9,405$

E-cigarette only

$4.0,574$

Cigarettes only

$9.5,1350$

$2.8,362$

Never used e-cigarette or cigarettes

Total, $N$

$79.7,11000$

13839

10th grade students

Initiated e-cigarette before cigarettes

$2.3,328$

Initiated e-cigarettes and cigarettes in same grade

$5.1,738$

Cigarettes before e-cigarettes

$7.0,1007$

E-cigarette only

$15.4,2211$

Cigarettes only

$3.6,494$

Never used e-cigarette or cigarettes

$66.7,9304$

Total, $N$

14082

12th grade students

Initiated e-cigarette before cigarettes

$2.2,83$

Initiated e-cigarettes and cigarettes in same grade

$5.1,191$

Cigarettes before e-cigarettes

$10.2,374$

E-cigarette only

$13.6,492$

Cigarettes only

$7.2,260$

Never used e-cigarette or cigarettes

$61.7,2273$

Total, $N$

3673

Public use weights are used to estimate percentages. Some percentages exceed $100 \%$ due to rounding. Unweighted sample sizes are provided in the tables. 
the initiation sequence of e-cigarette use and cigarette smoking among all respondents (ie, 8th, 10th, and 12th graders) were as follows: (1) e-cigarette use before cigarette smoking (1.7\%); (2) e-cigarette use and cigarette smoking in the same grade (4.2\%); (3) cigarette smoking before e-cigarette use (6.1\%); (4) e-cigarette use only (12.6\%); (5) cigarette smoking only (3.6\%); and (6) no e-cigarette use or cigarette smoking (71.8\%). Among those who reported both e-cigarette use and cigarette smoking, those who initiated cigarette smoking before e-cigarette use made up the largest proportion, while those who initiated e-cigarette use before cigarette smoking made up the smallest proportion across the 8th, 10th, and 12th grade samples.

Next, we examined the prevalence of substance use behaviors (ie, drunkenness, marijuana use, nonmedical prescription drug use, and other illicit drug use) as a function of initiation sequence of e-cigarette use and cigarette smoking (Table 2). Among the overall sample, the prevalence of all substance use behaviors was highest among those who reported a history of both e-cigarette use and cigarette smoking (regardless of initiation pattern), followed by those who reported cigarette smoking alone, e-cigarette use alone, and the lowest among adolescents who never engaged in e-cigarette use or cigarette smoking. No substantial differences in substance use behaviors were found between different initiation patterns among dual users. Those who indicated cigarette smoking alone had higher prevalence rates of different types of substance use (except for lifetime nonmedical prescription drug use). These patterns in the prevalence rates of substance use were similar within each grade cohort of 8 th, 10th, and 12 th graders.

The multivariate logistic regression analyses presented in Table 3 confirmed the bivariate analyses (Table 2). The adjusted odds ratios (AORs) of all lifetime substance use behaviors were significantly greater among those who reported any lifetime history of e-cigarette use or cigarette smoking compared with the odds for those who reported never using e-cigarettes or cigarette smoking (Table 3; post hoc comparisons were conducted but not presented in Table 3). For example, the adjusted odds of lifetime marijuana use among all respondents (ie, the combined sample of 8th, 10th, and 12th graders) who reported cigarette smoking before e-cigarette use were over sixty-five times greater than those who never reported e-cigarette use or cigarette smoking $(\mathrm{AOR}=65.8,95 \%$ confidence interval $[\mathrm{CI}]=55.5, \quad 78.0)$, after statistically controlling for sociodemographic characteristics including grade, race/ ethnicity, parental education, geographic region, metropolitan statistical area, and college plans. Additionally, the same pattern shown in Table 2 remains: the group with the highest odds of substance use were dual users (regardless of initiation pattern), followed by cigarette only smokers, e-cigarette only users, and lowest among adolescents who never engaged in ecigarette use or cigarette smoking. The only nonsignificant difference was between cigarette only users and e-cigarette only users who indicated nonmedical prescription drug use (note that these patterns hold within each grade cohort).

Table 4 provides the results assessing lifetime substance use behaviors when accounting for grade of initiation of e-cigarette use. Among lifetime e-cigarette users for 8th, 10th, and 12th graders, the prevalence of lifetime drunkenness, marijuana use, nonmedical prescription drug use, and other illicit drug use was higher among early initiators of e-cigarettes, when accounting for socio-demographic characteristics and initiation patterns for e-cigarette and cigarette use (see Table 4). Additionally, dual users (regardless of of initiation pattern) had higher odds of substance use when compared to e-cigarette only users across each grade level.

The results indicate two important findings. First, regardless of initiation sequence, earlier initiation of ecigarette use increases the odds of substance use. Second, regardless of whether e-cigarette use came before cigarette smoking or vice versa, the odds of engaging in substance use among dual users are still substantially higher when compared with e-cigarette only users (irrespective of when dual use was first initiated via e-cigarette use).

We conducted additional analyses to examine the initiation of e-cigarette use and all other substance use behaviors (including cigarette smoking, alcohol, smokeless tobacco, marijuana, inhalants, cocaine, crack, LSD, other hallucinogens/psychedelics, heroin, nonmedical prescription opioids, nonmedical prescription stimulants, or nonmedical prescription tranquilizers) among all respondents (results not shown). Among 8th, 10th, and 12th graders, the initiation patterns were as follows: (1) e-cigarette use before other substance use (1.7\%); (2) e-cigarette use and other substance use in the same grade $(6.9 \%)$; (3) other substance use before ecigarette use (13.6\%); (4) e-cigarette use only (3.4\%); (5) cigarette smoking only (20.5\%); and (6) no e-cigarette or cigarette smoking $(53.9 \%)$. Notably, the most prevalent initiation sequence of e-cigarette use involved initiating other substances before e-cigarettes, especially among older adolescents in 12th grade $(21.5 \%)$ relative to 10th grade students $(16.4 \%)$ and 8 th grade students $(8.7 \%)$.

\section{DISCUSSION}

Our findings provide valuable new information that US adolescents who reported both e-cigarette use and cigarette smoking were more likely to initiate cigarette smoking before e-cigarette use. The findings of the present study provide additional evidence that initiation sequence of e-cigarette use and cigarette smoking are associated with a wide range of other substance use behaviors, usually alcohol or marijuana use. The present study found that e-cigarette (only) users were at significantly lower risk than dual users and these findings are consistent with previous studies that found similar results for a wide range of problem behaviors and psychiatric symptoms. ${ }^{10,11,22,23}$ Notably, when adolescents engage in e-cigarette use in conjunction with cigarette 


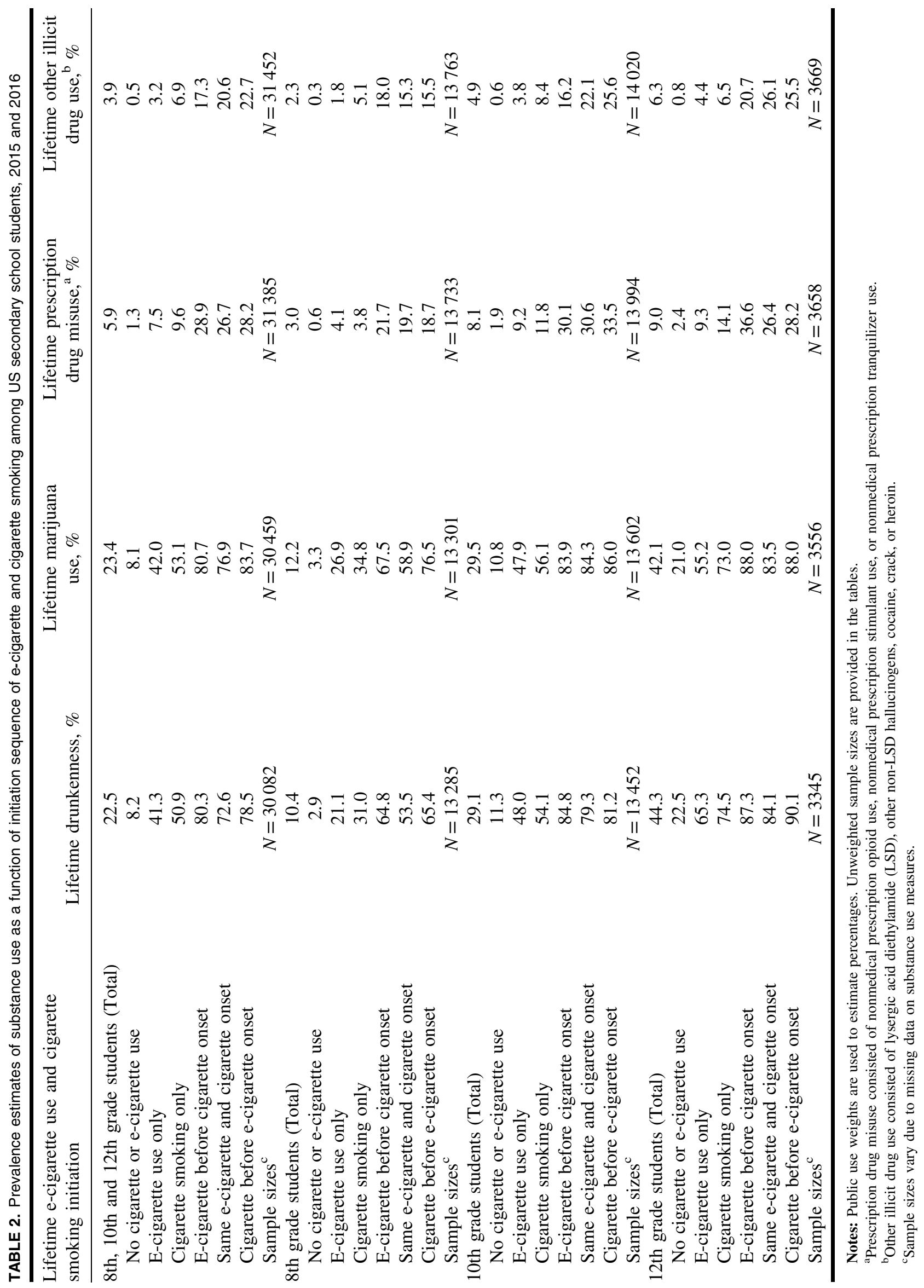




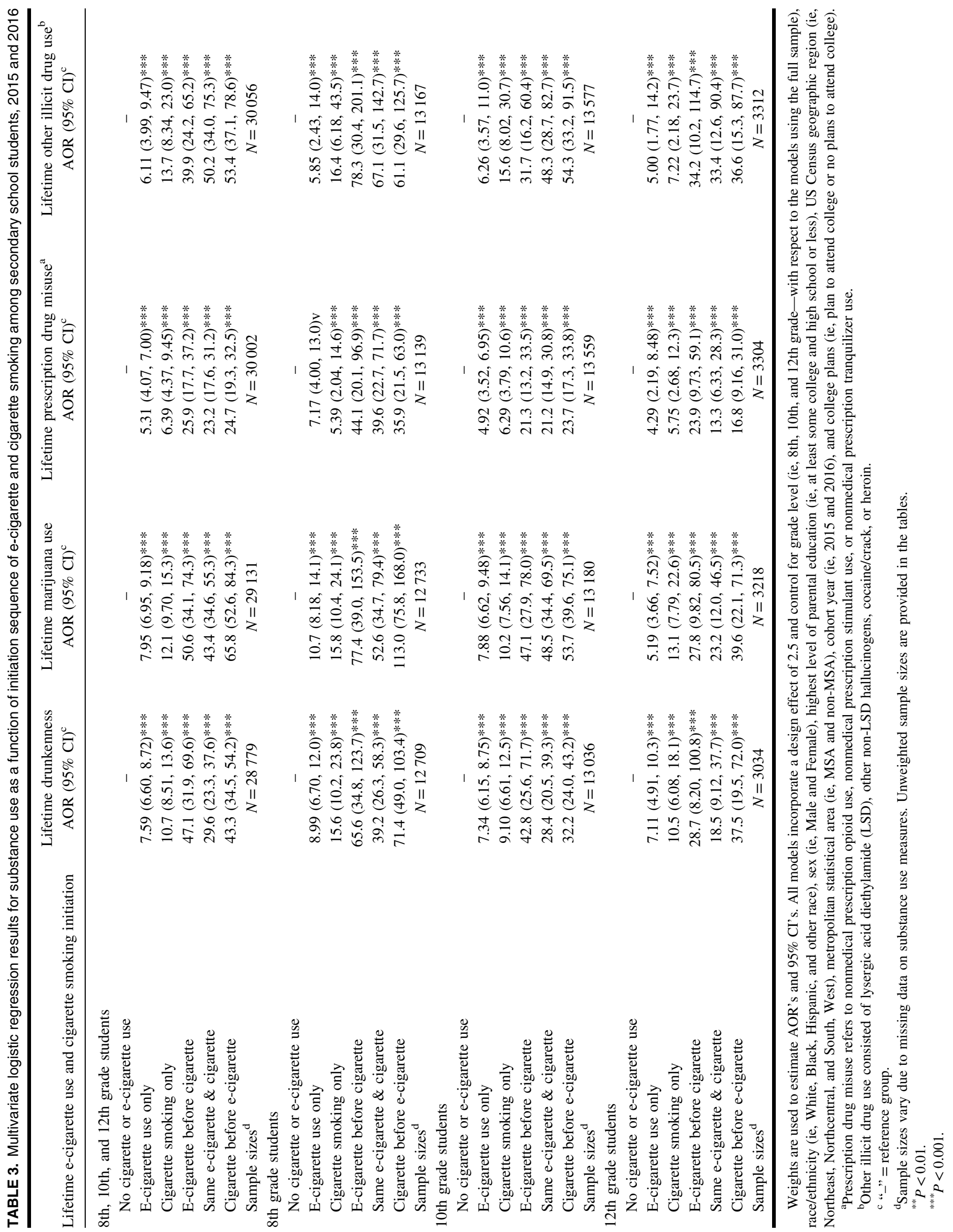




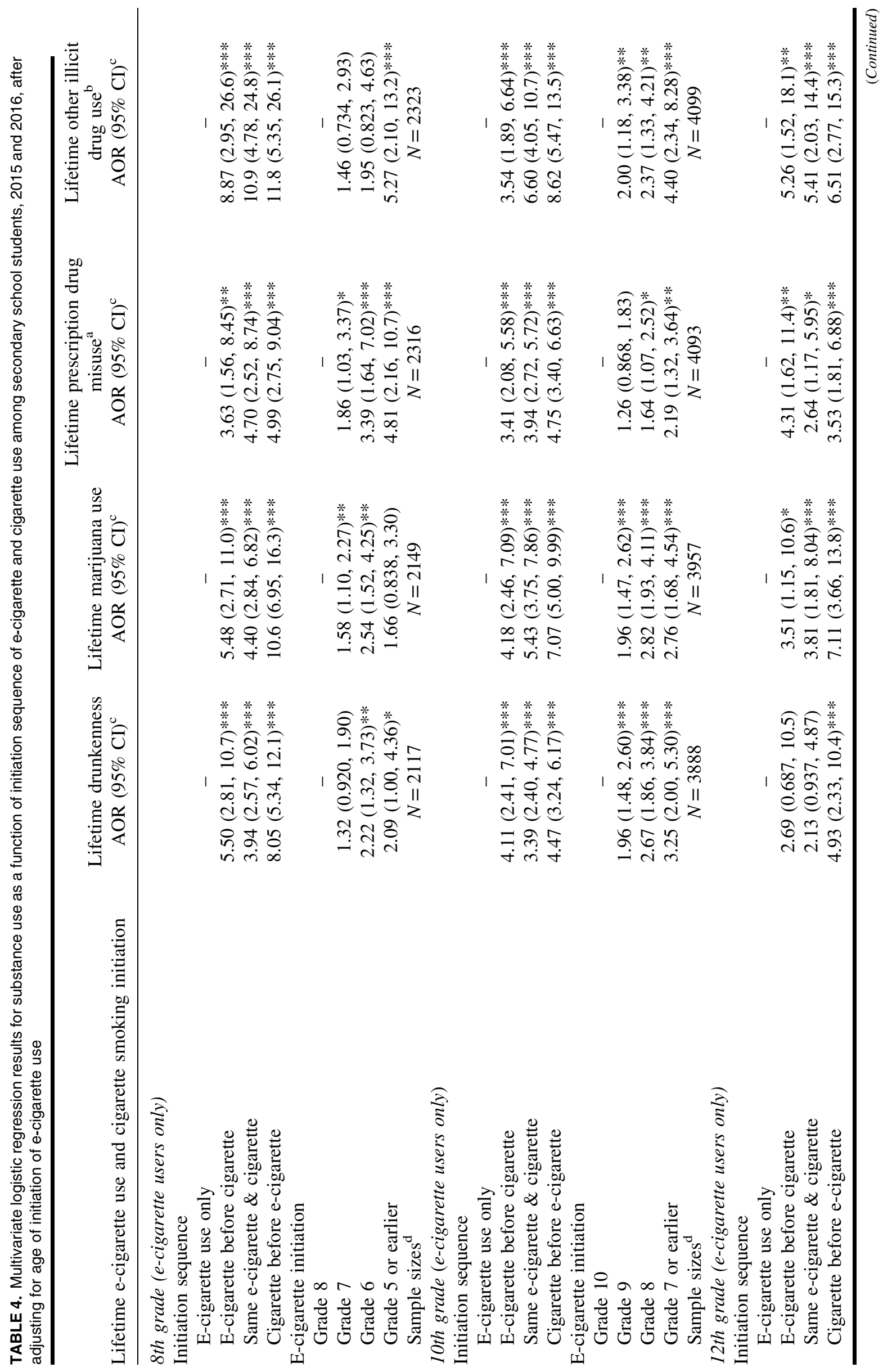


smoking, it often signals a form of polysubstance use that includes alcohol and marijuana.

The findings of the current study indicate that adolescents, who report a history of both e-cigarette use and cigarette smoking (regardless of temporal order of initiation), should be considered at high risk for other substances use behaviors and substance-related problems. Indeed, we found that over $80 \%$ of high-school students (10th and 12th grade) with a history of both e-cigarette use and cigarette smoking had also used marijuana and had been drunk. These findings indicate the need for long-term prospective longitudinal studies to examine these high-risk subgroups. The current study extends the well-established finding that the use of one substance often cooccurs with substance use and other problem behaviors. ${ }^{24}$ Indeed, there is growing evidence that adolescent dual users should be targeted for smoking cessation, substance use assessment, and screening for multiple problem behaviors. ${ }^{24}$

The relationship between early onset of drinking and other drug use with the development of subsequent substance-related problems has been well-established. ${ }^{25,26}$ The present study extends this earlier work indicating that early onset of ecigarettes was significantly associated with increased odds of heavy drinking, marijuana use, prescription drug misuse, and other illicit substance use behaviors relative to those who initiated later e-cigarette use as well as those who did not use e-cigarettes. These findings were present across all three age groups and advance our knowledge about the important role of early onset of e-cigarette use in predicting cigarette smoking and other substance use behaviors. Recent research found that adolescents with impaired self-regulation and behavioral impulsivity were more likely to report earlier onset of ecigarette use and more frequent e-cigarette use. ${ }^{27}$ The present study also found evidence that early onset of e-cigarettes, when adjusting for e-cigarette use only and dual use, was significantly associated with increased odds of engaging in other types of substance use. Other studies show that adolescents who engage in e-cigarette use only are at greater risk for other types of substance use, however, these studies have not fully considered the role of early onset vs late onset of e-cigarettes relative to dual users across multiple age groups. ${ }^{10,11,22,23,28}$ The results from this study extend prior work and suggest that earlier initiation of e-cigarette use remains a substantial risk factor for other types of substance use-even for this lower risk group of e-cigarette only users. Taken together, these findings indicate that adolescents who engage in early onset of e-cigarette use ( $e$ cigarette use only or dual use) should be assessed for substance-related problems based on their increased risk for polysubstance use. ${ }^{29}$ Moreover, the findings of the present study indicate that prospective studies examining the association between e-cigarette use and cigarette smoking must adequately account for prior history of substance use behaviors, especially among older adolescents.

\section{Strengths and Limitations}

The current study contains strengths and limitations that should be weighed carefully when considering the implications 
of the findings. One major strength of the current study is the large nationally representative sample of US secondary school students enrolled in public and private schools with a diverse range of sociodemographic characteristics. The large national sample permitted us to examine important subgroups as a function of initiation of e-cigarette use and cigarette smoking (eg, e-cigarette use after cigarette smoking). Despite these strengths, this study has limitations associated with large-scale school-based survey research using self-administered surveys and retrospective assessment, including nonresponse bias and missing data. First, some important subgroups do not participate in the MTF study including adolescents who are home-schooled, dropped out of school, and are absent on the day of data collection. ${ }^{2,30}$ Secondary school students who are home-schooled are less likely to engage in substance use behaviors while those who drop out or who are often absent from school are more likely to engage in substance use and other problem behaviors. ${ }^{2,30}$ Second, the MTF study did not assess some variables that have been shown to be associated with early onset of substance use behaviors (eg, family psychiatric history). Notably, sensation-seeking measures were available for 8th and 10th grade students and additional analyses revealed the significant multivariable findings in Table 3 and 4 remained significant after controlling for sensation-seeking as a covariate. Third, although self-report data in the MTF study have been found to be reliable and valid, studies on adolescents suggest that misclassification and under-reporting of sensitive behaviors such as substance use does occur. ${ }^{31}$ Finally, given the cross-sectional design of the MTF, the current study may suffer from reporting bias due to the retrospective nature of the questions. While this may limit the validity of the responses, the large sample of adolescents makes it possible to construct categories of initiation to assess substance use behaviors across these different groups. The data were from 2015 and 2016 and do not capture recent products such as Juul and Suorin, and marijuana legalization which all could have an impact on initiation patterns. Furthermore, the cross-sectional retrospective nature of this study is precisely why more prospective longitudinal studies should be undertaken with more recent cohorts of adolescents to examine whether the associations found in the present study hold over time.

\section{CONCLUSION}

Public health experts recognize that adolescents' e-cigarette use is understudied, and there is a call for more rigorous research. ${ }^{3}$ The present study provides timely insights into adolescents' use of e-cigarettes within a larger context of substance use. With its national sample and robust substance use measures, this study fills an important gap by contributing new knowledge and providing directions for future policy and research to address this important public health need.

Undeniably, the benefits and harms of e-cigarettes remain unclear. E-cigarettes emerged on the United States market in
2009 and we do not know enough about long-term consequences of e-cigarette use generally, nor adolescent e-cigarette use specifically. In the future, researchers can build on these findings and focus on longer-term consequences, use trajectories among sub-groups, cohort effects, and health consequences of adolescent e-cigarette use while accounting for the important role of other substance use.

Funding for this study was supported by research grants from the National Cancer Institute (R01CA203809) and National Institute on Drug Abuse (R01DA031160 and R01DA044157) at the National Institutes of Health. The Monitoring the Future data were collected under research grant R01DA001411. The content is solely the responsibility of the authors and does not necessarily represent the official views of the National Cancer Institute, National Institute on Drug Abuse, or the National Institutes of Health. The sponsors had no additional role in the design and conduct of the study; collection, management, analysis, and interpretation of the data; and preparation, review, or approval of the manuscript. There was no editorial direction or censorship from the sponsors. The authors would like to thank the National Addiction and HIV Data Archive Program for providing access to these data. The authors would like to thank the respondents and school personnel for their participation in the study. The authors would like to thank summer high school research intern Ms Riley Dickinson for her help with the literature review, tables, and suggestions to a previous version of this manuscript. The authors wish to thank undergraduate research assistant Ms Kara Dickinson who assisted in the proof-reading of the manuscript.

\section{Declaration of Interest}

The authors report no conflicts of interest. The authors alone are responsible for the content and writing of this paper.

\section{REFERENCES}

1. Jamal A, Gentzke A, Hu SS, et al. Tobacco use among middle and high school students-United States, 2011-2016. MMWR Morb Mortal Wkly Rep. 2017;66(23):597-603.

2. Miech RA, Schulenberg JE, Johnston LD, Bachman JG, O'Malley PM, Patrick ME National Adolescent Drug Trends in 2018. Monitoring the Future, Ann Arbor, MI; 2018. http://www.monitoringthefuture.org. Accessed Jan 22, 2019.

3. National Academies of Sciences, Engineering, and Medicine. Public health consequences of e-cigarettes. Washington, DC: The National Academies Press; 2018.

4. Singh T, Arrazola RA, Corey CG, et al. Tobacco use among middle and high school students--United States, 2011-2015. MMWR Morb Mortal Wkly Rep. 2016;65(14):361-367.

5. Barrington-Trimis JL, Urman R, Berhane $\mathrm{K}$, et al. E-cigarettes and future cigarette use. Pediatrics. 2016;138:e20160379.

6. Leventhal AM, Strong DR, Kirkpatrick MG, et al. Association of electronic cigarette use with initiation of combustible tobacco product smoking in early adolescence. JAMA. 2015;314(7):700-707.

7. Miech R, Patrick ME, O'Malley PM, Johnston LD. E-cigarette use as a predictor of cigarette smoking: results from a 1-year follow-up of a 
national sample of 12th grade students. Tob Control. 2017;26(e2): e106-e111.

8. Dai H, Hao J. Electronic cigarette and marijuana use among youth in the United States. Addict Behav. 2017;2017(66):48-54.

9. Hughes K, Bellis MA, Hardcastle KA, et al. Associations between ecigarette access and smoking and drinking behaviours in teenagers. BMC Pub Health. 2015;15:244.

10. Kristjansson AL, Mann MJ, Sigfusdottir ID. Licit and illicit substance use by adolescent e-cigarette users compared with conventional cigarette smokers, dual users, and nonusers. J Adolesc Health. 2015;57(5): $562-564$.

11. McCabe SE, West BT, Veliz P, Boyd CJ. E-cigarette use, cigarette smoking, dual use, and problem behaviors among U.S. adolescents: results from a national survey. J Adolesc Health. 2017;61(2):155-162.

12. Primack BA, Soneji S, Stoolmiller M, Fine MJ, Sargent JD. Progression to traditional cigarette smoking after electronic cigarette use among US adolescents and young adults. JAMA Pediatr. 2015;169(11):1018-1023.

13. Unger JB, Soto DW, Leventhal A. E-cigarette use and subsequent cigarette and marijuana use among Hispanic young adults. Drug Alcohol Depend. 2016;163:261-264.

14. Watkins SL, Glantz SA, Chaffee B. Association of noncigarette tobacco product use with future cigarette smoking among youth in the Population Assessment of Tobacco and Health (PATH) Study, 20132015. JAMA Pediatr. 2018;172(2):181-187.

15. Wills TA, Knight R, Sargent JD, Gibbons FX, Pagano I, Williams RJ. Longitudinal study of e-cigarette use and onset of cigarette smoking among high school students in Hawaii. Tob Control. 2017;26(1):34-39.

16. Audrain-McGovern J, Stone MD, Barrington-Trimis J, Unger JB, Leventhal AM. Adolescent e-cigarette, hookah, and conventional cigarette use and subsequent marijuana use. Pediatrics. 2018;142(3):e20173616.

17. Collaco JM, Drummond MB, McGrath-Morrow SA. Electronic cigarette use and exposure in the pediatric population. JAMA Pediatr. 2015; 169(2):177-182.

18. Wills TA, Gibbons FX, Sargent JD, Schweitzer RJ. How is the effect of adolescent e-cigarette use on smoking onset mediated: a longitudinal analysis. Psychol Addict Behav. 2016;30(8):876-886.

19. Soneji S, Barrington-Trimis JL, Wills TA, et al. Association between initial use of e-cigarettes and subsequent cigarette smoking among adolescents and young adults: a systematic review and meta-analysis. JAMA Pediatr. 2017;171(8):788-797.

20. Miech RA, O'Malley PM, Johnston LD, Patrick ME. E-cigarettes and the drug use patterns of adolescents. Nicotine Tob Res. 2016;18(5):654-659.

21. West BT, McCabe SE. Incorporating complex sample design effects when only final survey weights are available. Stata J. 2012;12(4):718-725.

22. Demissie Z, Jones S, Clayton H, King B. Adolescent risk behaviors and use of electronic vapor products and cigarettes. Pediatrics. 2017; 139(2):e20162921. 2017

23. Leventhal AM, Strong DR, Sussman S, et al. Psychiatric comorbidity in adolescent electronic and conventional cigarette use. J Psychiatr Res. 2016;73:71-78.

24. Biglan A, Brennan PA, Foster SL, Holder HD Helping adolescents at risk: prevention of multiple problem behaviors. New York, NY: Guilford Press; 2014.

25. Grant BF, Dawson DA. Age at onset of drug use and its association with DSM-IV drug abuse and dependence: results from the National Longitudinal Alcohol Epidemiological Survey. $J$ Subst Abuse. 1998;10:163-173.

26. Hawkins JD, Graham JW, Maguin E, Abbott R, Hill KG, Catalano RF. Exploring the effects of age of alcohol use initiation and psychosocial risk factors on subsequent alcohol misuse. J Stud Alcohol. 1997;58(3): 280-290.

27. Bold KW, Kong G, Camenga DR, et al. Trajectories of e-cigarette and conventional cigarette use among youth. Pediatrics. 2018;141:e20171832.

28. McCabe SE, West BT, McCabe VV. Associations between early onset of e-cigarette use and cigarette smoking and other substance use among U.S. adolescents: results a national survey. Nicotine Tob Res. 2018; 20(8):923-930.

29. Silveira ML, Conway KP, Green VR, et al. Longitudinal associations between youth tobacco and substance use in waves 1 and 2 of the Population Assessment of Tobacco and Health (PATH) Study. Drug Alcohol Depend. 2018;191:25-36.

30. Vaughn MG, Salas-Wright CP, Kremer KP, Maynard BR, Roberts G, Vaughn S. Are homeschooled adolescents less likely to use alcohol, tobacco, and other drugs? Drug Alcohol Depend. 2015;155:97-104.

31. Harrison L, Hughes A. The validity of self-reported drug use: improving the accuracy of survey estimates. NIDA Rese Monogr. 1997;167:1-16. 